\title{
A Clinical Dosimetry Analysis of Total Body Irradiation for Leukemia Patients
}

\author{
Lanchun Lu*, Jonathan Filippi, Akshi Patel, Nilendu Gupta, Michael Weldon, Jeffery Woollard, \\ Ahmet Ayan, Yi Rong, Douglas Martin, Christopher Pelloski, Meng Xu Welliver \\ Department of Radiation Oncology, The Ohio State University, Columbus, USA \\ Email: ${ }^{1}$ lu.281@osu.edu
}

Received October 7, 2013; revised November 6, 2013; accepted December 3, 2013

Copyright (C) 2014 Lanchun Lu et al. This is an open access article distributed under the Creative Commons Attribution License, which permits unrestricted use, distribution, and reproduction in any medium, provided the original work is properly cited. In accordance of the Creative Commons Attribution License all Copyrights (C) 2014 are reserved for SCIRP and the owner of the intellectual property Lanchun Lu et al. All Copyright @ 2014 are guarded by law and by SCIRP as a guardian.

\section{ABSTRACT}

Background and Purpose: To perform a retrospective in vivo dosimetry study of 129 total body irradiation (TBI) on leukemia and bone marrow transplant patients treated in our clinic from 2008 to 2011 and to find out if there is any indication of the necessity of developing a new efficient TBI approach. Materials and Methods: The in vivo dosimetry data of 129 patients treated with TBI between 2008 and 2011 were retrieved from the database and analyzed. These patients were mostly treated with the regime of a single fraction or 6 fractions with some exceptions of 8-fraction or 2-fraction treatments depending on the protocols that were applied. For every fraction of treatment, 10 pairs of diode dosimeters were used to monitor the doses to the midline of head, neck, arms, mediastinum, left lung, right lung, umbilicus, thigh, knee, and ankle for both AP and PA fields. The doses to the midline of the above body parts were considered to be the average of the AP and PA readings of each diode pair. Dose deviation from the prescribed value for each body part was studied by plotting the histogram of the frequency versus deviation and comparing this with the dose delivered to the midline of the umbilicus to where the dose was prescribed. The correlation of dose deviation to body part thickness was also studied. By studying the dose deviations, we can find the uniformity of general dose distributions for conventional TBI treatments. Results: The retrospective dosimetry study of the 129 TBI patient treatments indicates that for most of the patients treated in our clinic, the doses received by different body parts monitored with in vivo dosimetry were within the window of $10 \%$ difference from the prescribed dose. The inhomogeneity of dose on different body parts could be manually improved by using compensators, but the method is cumbersome and time consuming. The dose deviation in many histograms ranging from about $-10 \%$ to $10 \%$ indicates some incongruity of dose distribution. This could be due to the method of using lead compensators for a manual dose adjustment which could not ideally compensate for different body thicknesses everywhere. Conclusions: The conventional TBI could give uniform dose to the major body parts under the online in vivo dosimetry monitoring at the level of $10 \%$, but the treatment procedure is cumbersome and time consuming. This implies the importance of developing a new and efficient TBI method by adopting modern radiation therapy technique.

\section{KEYWORDS}

Total Body Irradiation (TBI); In Vivo Dosimetry; Lung Block; Compensators; Patient Setup; Treatment Planning

\section{Introduction}

Total body irradiation (TBI) has been frequently used in many clinics for leukemia patients prior to a bone marrow transplant to eradicate tumor cells and suppress pa-

\footnotetext{
"Corresponding author.
}

tients' immune system to enhance the chance of a successful marrow graft. The most common pretreatment conditioning is a combination of chemotherapy and TBI. The conventional and the most often-used TBI techniques in clinics in the past compared to now may vary but have always been intended to give a uniform dose of 
radiation to the whole body of a patient and limit the dose to the lungs. The process can destroy cancer cells and reduce the reaction of the body's immune system so that it will not attack the donor's cells during the transplant.

TBI treatment has a long history which started as early as 1932 [1]. Since then, the TBI treatment technique has evolved with the development of treatment units starting from X-ray tube machines to the radioactive source machines like Cobalt-machines, and then to modern-day high energy linear accelerators. The treatment techniques also varied from machine to machine and facility to facility. Of these conventional TBI techniques, the most often used is the lateral decubitus position. This treatment begins with an in-treatment room clinical setup/ simulation, with radiographs taken at the extended treatment distance for lung localization. Typically, an X-ray simulator or CT-simulator is not used for this setup except for making lung blocks, and the treatment planning consists of hand calculations. First, the ray line thicknesses of the patient at different anatomical regions are measured manually, with the patient in the treatment position (lateral decubitus in our institution). This includes the thicknesses of head, neck, mid-mediastinum, left and right lungs, umbilicus, thigh, knee, ankle, and arms. Then $\mathrm{X}$-ray radiographs are taken for the AP and PA positions in which the treatment will be administered. These radiographs are also used for fabrication of lung blocks (which usually are made of cerrobend) that are used to limit the dose to the left and right lungs during the treatment. This ultimately reduces the incidence of radiation pneumonitis. Based on these measurements, the dose to the patient is manually calculated and the thicknesses of lead sheets used to compensate the doses delivered to different anatomical areas during the treatment are determined. The radiation dose is prescribed at the midline of the umbilicus. With the thickness of umbilicus and the prescribed dose, the monitor units (MUs) for AP and PA beams are calculated. The most commonly used treatment regime for TBI is usually prescribed a total dose of $12 \mathrm{~Gy}$ to the body while the lungs are specified to receive no more than 10 Gy for 6 fractions - 2 Gy/fraction bidaily for 3 days, with variations depending on the chemotherapy regimen used. The treatment is conducted at extended distances (typically with a source-to-skin distance of $400 \mathrm{~cm}$ to $500 \mathrm{~cm}$ for adults), to accomplish a dose rate of 6 - $12 \mathrm{cGy} / \mathrm{min}$.

Different clinics may have different methods of patient setup [2,3]. For example, one method uses bilateral technique which allows the patient to be seated with the back supported and legs bent. Another method allows the patient to be in a standing position with lung blocks and compensators mounted on an acrylic plate placed in front. Although these techniques are different in the details, they are all aimed to give a uniform dose to the patient body and all manually use compensators or lung blocks to compensate doses to different body parts such as the lung and brain. Most of these TBI procedures apply in vivo patient dosimetry during the treatment to monitor the dose received by the patient. We can call these methods the conventional TBI.

Nowadays the majority of clinics still use the conventional TBI techniques mentioned above except for a few trials [4-6], which simply apply AP and PA fields without using Multileaf Collimator (MLC) to modulate the beams delivering prescribed doses to different body parts. 3D treatment plan technique is also not used for the treatments, which means that the detail dose distributions are not available for evaluating the treatments.

Although conventional TBI procedures have been existing in clinics for many years, there is not a systematic study to show the dosimetric results from a large patient samples. In this paper, we report the conventional TBI technique used in our clinic and a retrospective dosimetry study of 129 patients retrieved from the database from 2008 to 2011. We will study: 1) the dose deviation from the prescribed value for each body part by plotting the histogram of the frequency versus deviation and comparing with the dose delivered to the midline of the umbilicus to where the dose was prescribed; 2) the correlation of dose deviation to body part thickness. By studying the dose deviations, we can find out the dose uniformity of a general dose distribution for conventional TBI treatments. We hope that the results from this retrospective study will provide us with supporting evidence to develop an automatic beam modulated TBI method using the modern advanced linear accelerator in our clinic.

\section{Methods and Materials}

Treatment parameters were retrieved from the treatment database and analyzed. Most of these patients were treated with one single fraction 200 cGy and 6 fractions for a total of 3 days (bi-daily), 1200 cGy (200 cGy/fraction), and others were treated with 8 fractions, 11 fractions, or 2 fractions, depending on which protocol was applied.

\subsection{Patient Setup, Simulation and Treatment Planning}

The technique used to treat these patients requires patients to be lying down on their sides, in a semi-squatting position, facing the linac gantry for AP fields, and facing away from the linac gantry for PA fields with a source surface distance (SSD) of $500 \mathrm{~cm}$. The patients were positioned with their knees bent and arms on the side on which they were lying brought up to at least a 90-degree angle from the body and tucked under their heads. The other arm was down by their side. Figure 1 shows the 


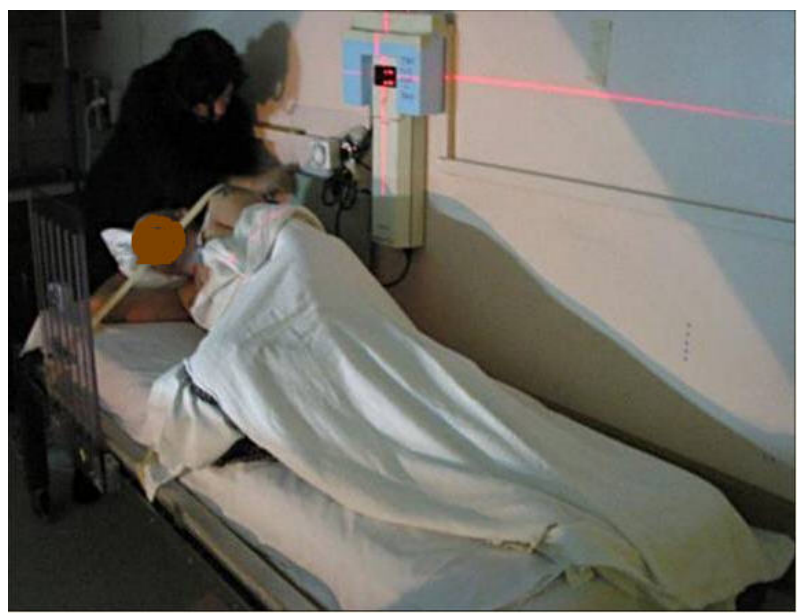

(a)

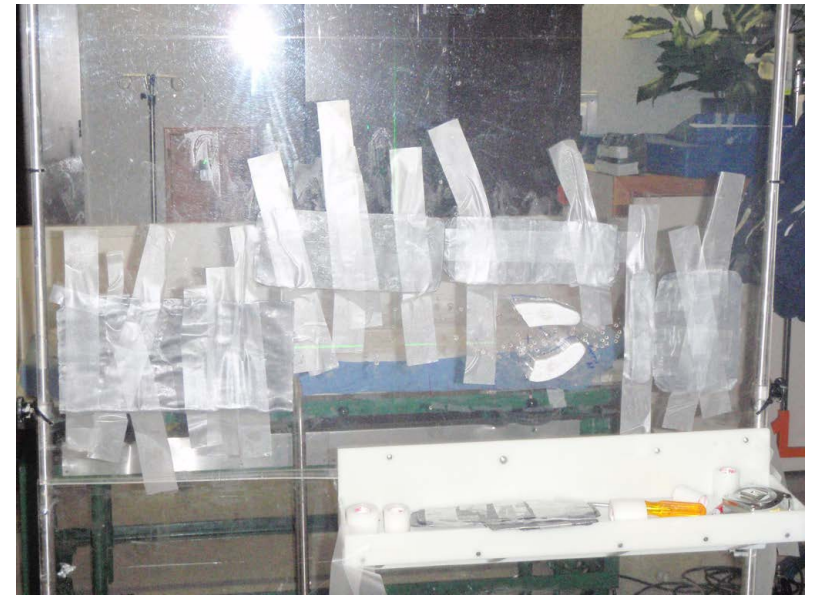

(b)

Figure 1. TBI setup: (a) Patient's position; (b) Lung block and compensators.

example setup of a patient. At the time of treatment simulation, patient was positioned on the special treatment table, at the furthest possible distance (usually SSD = $500 \mathrm{~cm}$, field size $40 \mathrm{~cm} \times 40 \mathrm{~cm}, 90^{\circ}$ collimator angle), to check for the adequacy of beam coverage using the light field. During this positioning check, the thicknesses of the head, neck, arm, mid-sternum, lungs, umbilicus, thighs, knees, feet, and ankles were determined and the x-ray computerized radiographs for lungs were taken which would be used for making lung blocks before the actual treatments. Pre-treatment physics dosimetry calculations were made based on the measurements above. An isocentric patient setup was assumed with a SSD (source-surface-distance) of $500 \mathrm{~cm}$ for the AP and PA Fields.

The output of the linear accelerator is checked each month for $1 \mathrm{cGy} / \mathrm{MU}$ at $100 \mathrm{~cm}$ and $10 \times 10 \mathrm{~cm}^{2}$ field size. The output of the machine for the AP and PA fields at the prescribed point was calculated as a product of inverse-square factor and the measured output at SSD =
$500 \mathrm{~cm}$. The Tissue Maximum Ratio (TMR) used in the Monitor Unit (MU) calculations was taken from the beam data book of the treatment unit. To reduce the dose to the lungs to a level of 1000 cGy, we used the halfvalue layer customized lung blocks, which were made of cerrobend, a low melting point alloy with about $83 \%$ lead density. The lung blocks were mounted on a plastic screen which was $300 \mathrm{~cm}$ away from the patients' umbilicus surface. The attenuation of the treatment beam due to the plastic screen was accounted for by a measured tray factor 0.980 .

The MU used for these treatments were determined based on the prescribed dose to the midline of a patient at the level of the umbilicus. The formula used is the following:

M.U.

$=($ Prescribed Dose $) /($ Output $\times$ TMR $\times$ Tray Factor $)$

\subsection{In Vivo Dosimetry and Online Dose Monitoring}

During each fraction of treatments, in vivo dosimetry was performed to monitor the dose received by different body parts. Lead compensators were used for arms, neck, thighs, knees, and ankles as needed to compensate the smaller separation of these organs and to make sure the dose was being uniformly delivered to each body part. Lead is used as a compensator because its high attenuation ability allows us to make the compensator thinner. Additionally, it is rather soft compared with other metals so it is easy to be cut and made into different shapes. The lead compensator is composed of different layers and sizes of lead sheets, and each lead sheet is $1 \mathrm{~mm}$ thick. The number of layers can be changed as needed for different fractions of treatment. Diode dosimeters were used for the in vivo dosimetry. The end-point of dose monitoring is to achieve all readings within $\pm 10 \%$ of the prescription dose and $<1000$ cGy for lungs. The thickness of lead compensators were adjusted as needed based on the online dose monitoring. The in vivo dosimetry was performed using the commercial product IVD Solution in vivo dosimetry system (Sun Nuclear Corporation, Melbourne, FL, USA) which includes a series of diode dosimeters, a controller, and software managing the diodes, which collect readings and convert them to doses. For this in vivo dosimetry system, we used 10 pairs of diodes to monitor doses to the body parts of head, neck, mediastinum, left and right lungs, umbilicus, thighs, knees, ankles, and arms. Each body part used a pair of diodes to monitor the entrance dose and exit dose [the diodes were labeled as AP (PA) and PA (AP) respectively for AP (PA) field]. The dose to midline was determined by the average of the entrance and exit doses. At the end of delivery of each field (AP or PA), the dose from the field was 
recorded and exported to an Excel file. The total dose to the midline plane was the sum of the doses from the AP and PA fields.

For a multi-fraction TBI treatment, in order to have the lung dose be close to but not exceed $1000 \mathrm{cGy}$, we had to use whole-number fractions with and without lung blocks. This was done using an Excel formula to find out the pattern of the best combination of fractions with blocks and fractions without blocks based on the in vivo dosimetry measurements of lung dose and umbilicus dose for the first few fractions (including fractions with blocks and without blocks). Table 1 shows an example of the method used to determine the pattern. Table 2 shows the final results of a 6 -fraction TBI treatment with a pattern of 3.5 fractions with lung blocks and 2.5 fractions without.

\subsection{Retrospective Dosimetry Analysis of $\mathbf{1 2 9}$ Patients' TBI Treatments}

These patients were mostly treated with the regime of a single fraction and 6-fraction with some exceptions of 8-fraction, 2-fraction, and 11-fraction treatments, depending on the protocols that were applied. For each patient, during every fraction of treatment, 10 pairs of diode dosimeters were used to monitor the doses to the midline of head, neck, arms, mediastinum, left lung, right lung, umbilicus, thighs, knees, and ankles for both AP and PA fields. The doses to the midlines of the above body parts were considered to be the average of the AP and PA readings of each diode pair. Based on the dose information recorded in the database, we investigated the dose deviation from the prescribed value for each body part by plotting histograms of frequency of deviation and comparing with the dose delivered to midline of umbilicus to where the dose was prescribed. The correlation of dose deviation to body part thickness was also studied. By studying the dose deviations, we found the uniformity of general dose distribution for conventional TBI treatments.

\section{Results}

\subsection{Dose Deviation from Prescription}

Figure 2 shows the dose deviation from the prescribed value for head, neck, arm, umbilicus, mediastinum, lung, thigh, knee, and ankle. The horizontal (x-) axis is for dose difference in percentage from the prescribed dose (except for the lung, which is the difference from 1000 cGy for multi-fraction treatments), and the vertical (y-) axis is for the frequency of a deviation (the number of patients who have that dose deviation). Each graph includes all the treatment regimes, color-coded, 1-fraction (40 patients), 2-fraction (3 patients), 6-fraction (66 patients), 8-fraction (16 patients), and 11-fraction (4 patients), among which 1-fraction and 6-fraction cases are the dominant TBI treatments in our clinic (40 and 66 respectively). From these figures, we found that overall, for all body parts, the dose deviation from the prescribed value is less than $10 \%$, although there are a few exceptions

Table 1. The pattern of fractions using lung blocks and without using lung blocks.

\begin{tabular}{cccc}
\hline Block & No Block & $\begin{array}{c}\text { Lung Dose } \\
\text { (\% of Rx ) }\end{array}$ & $\begin{array}{c}\text { Estimated Lung } \\
\text { Dose (cGy) }\end{array}$ \\
\hline 2 & 4 & $96.8 \%$ & 1161 \\
2.5 & 3.5 & $91.8 \%$ & 1101 \\
3 & 3 & $86.7 \%$ & 1041 \\
3.5 & 2.5 & $\mathbf{8 1 . 7 \%}$ & $\mathbf{9 8 1}$ \\
4 & 2 & $76.7 \%$ & 921 \\
4.5 & 1.5 & $71.7 \%$ & 860 \\
5 & 1 & $66.7 \%$ & 800 \\
\hline
\end{tabular}

Table 2. In-vivo dosimetry results for a 6-fraction TBI treatment.

\begin{tabular}{|c|c|c|c|c|c|c|c|c|c|}
\hline Fraction & Head & Neck & Mediastinum & Lung & Umbilicus & Thigh & Knee & Ankle & Arm \\
\hline 1 & 223 & 216 & 234 & 136 & 227 & 218 & 190 & 212 & 193 \\
\hline 3 & 195 & 194 & 218 & 112 & 208 & 183 & 184 & 196 & 152 \\
\hline 4 & 218 & 197 & 184 & 173 & 202 & 188 & 195 & 221 & 148 \\
\hline 5 & 191 & 191 & 184 & 113 & 196 & 181 & 195 & 168 & 164 \\
\hline 6 & 192 & 190 & 195 & 214 & 200 & 198 & 213 & 196 & 231 \\
\hline Total & 1201 & 1184 & 1248 & 972 & 1226 & 1171 & 1176 & 1202 & 1119 \\
\hline$\%$ of RX & $100 \%$ & $99 \%$ & $104 \%$ & $81 \%$ & $102 \%$ & $98 \%$ & $98 \%$ & $100 \%$ & $93 \%$ \\
\hline
\end{tabular}




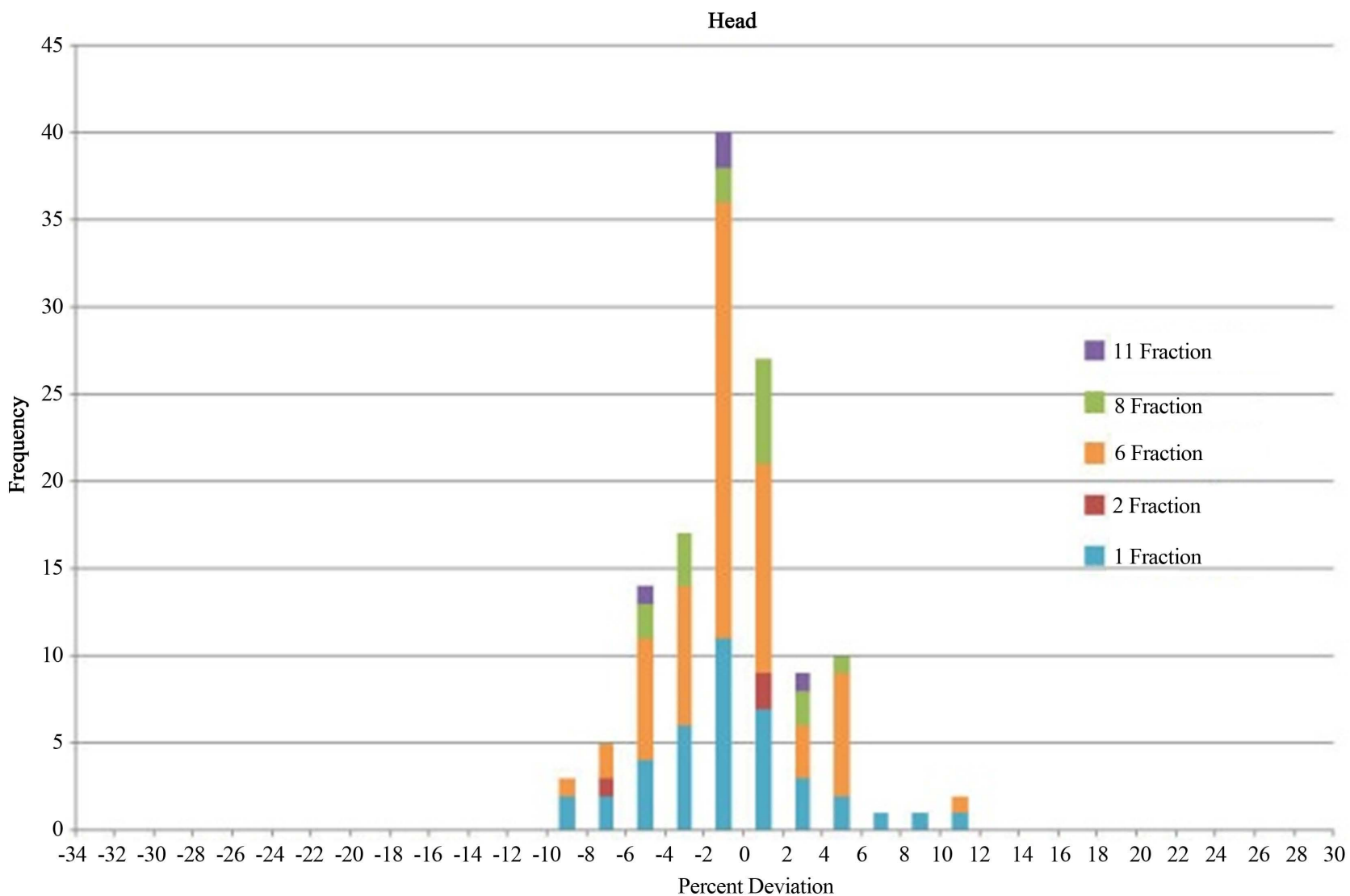

Neck

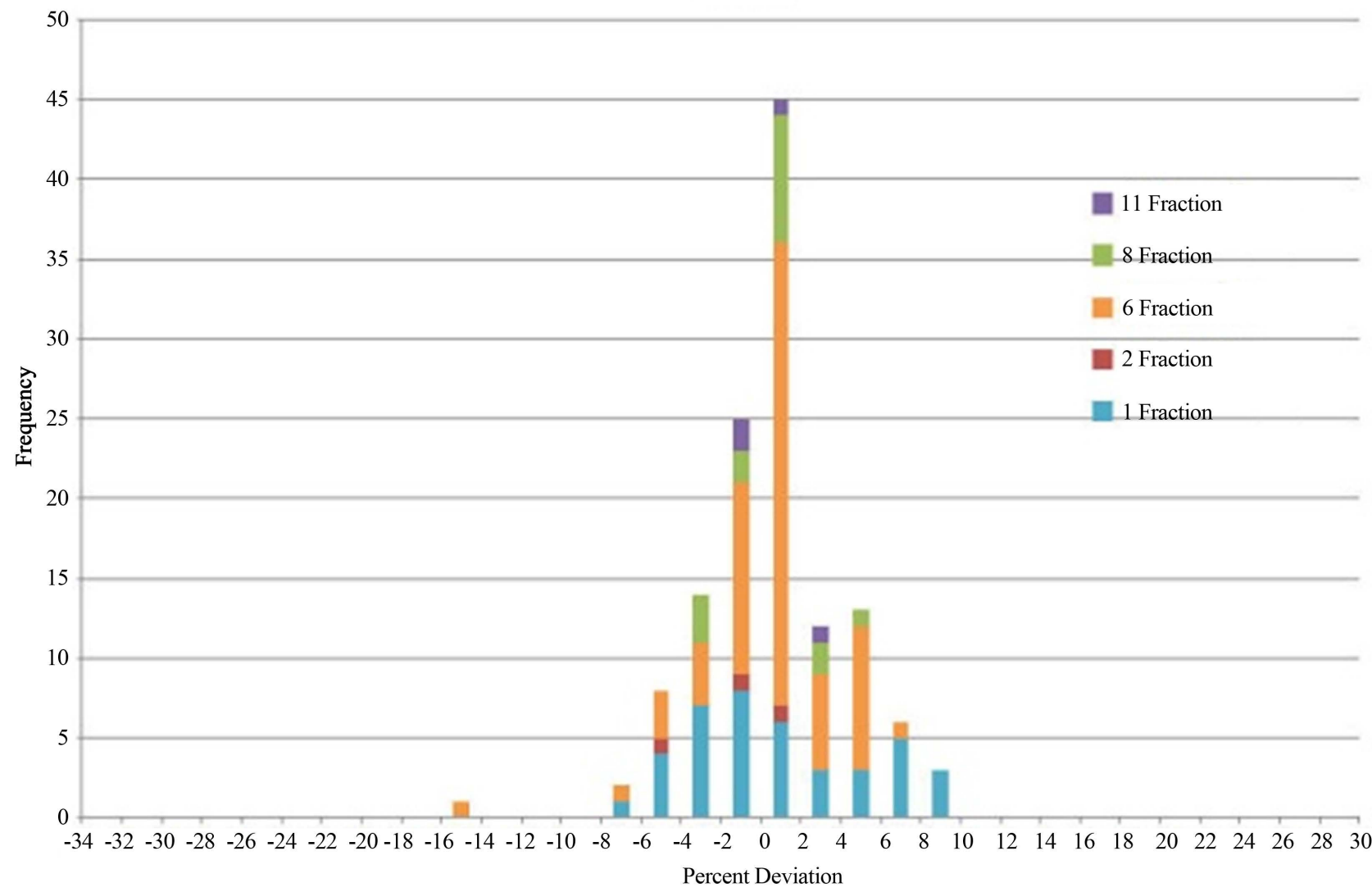




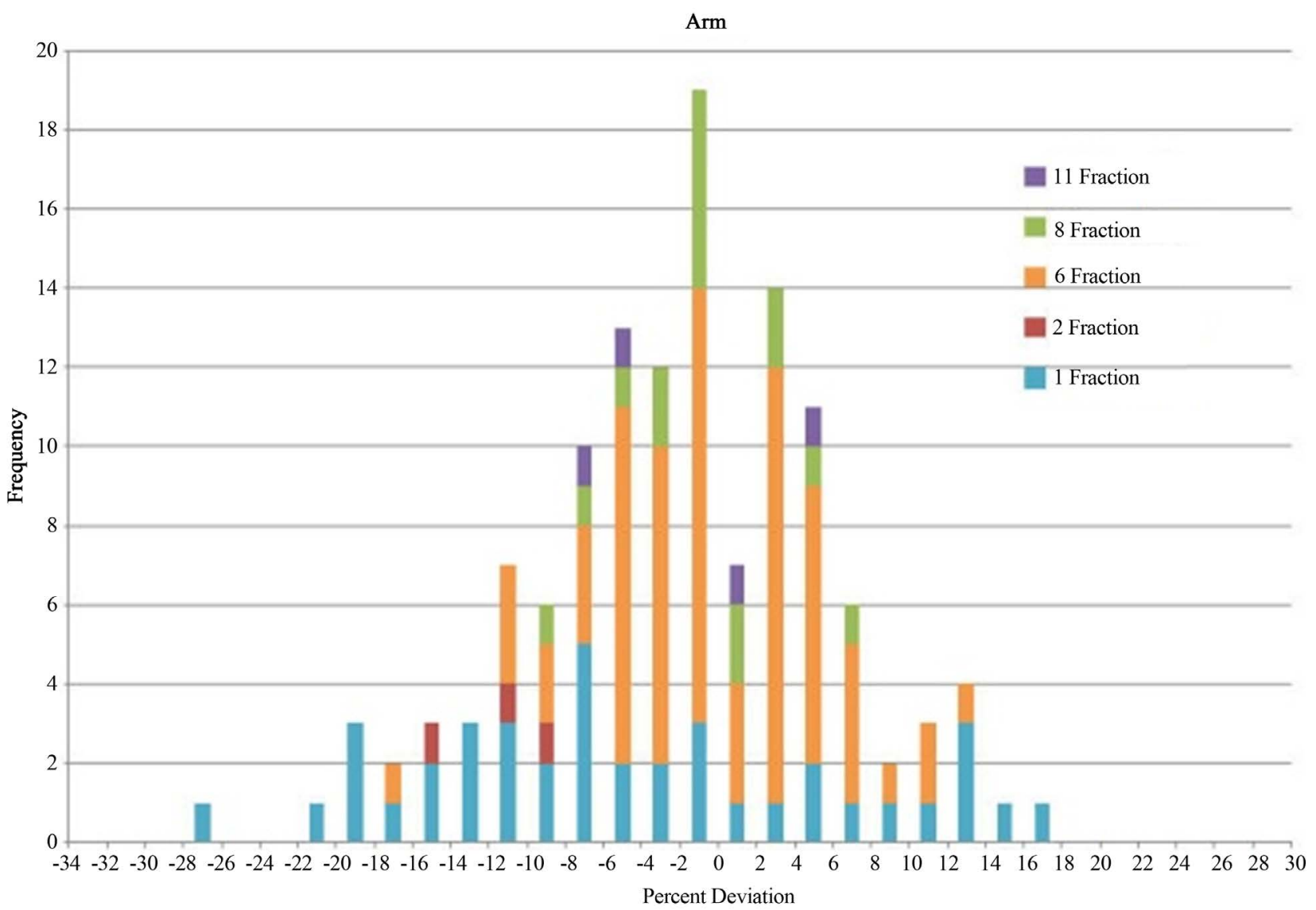

Umbilicus

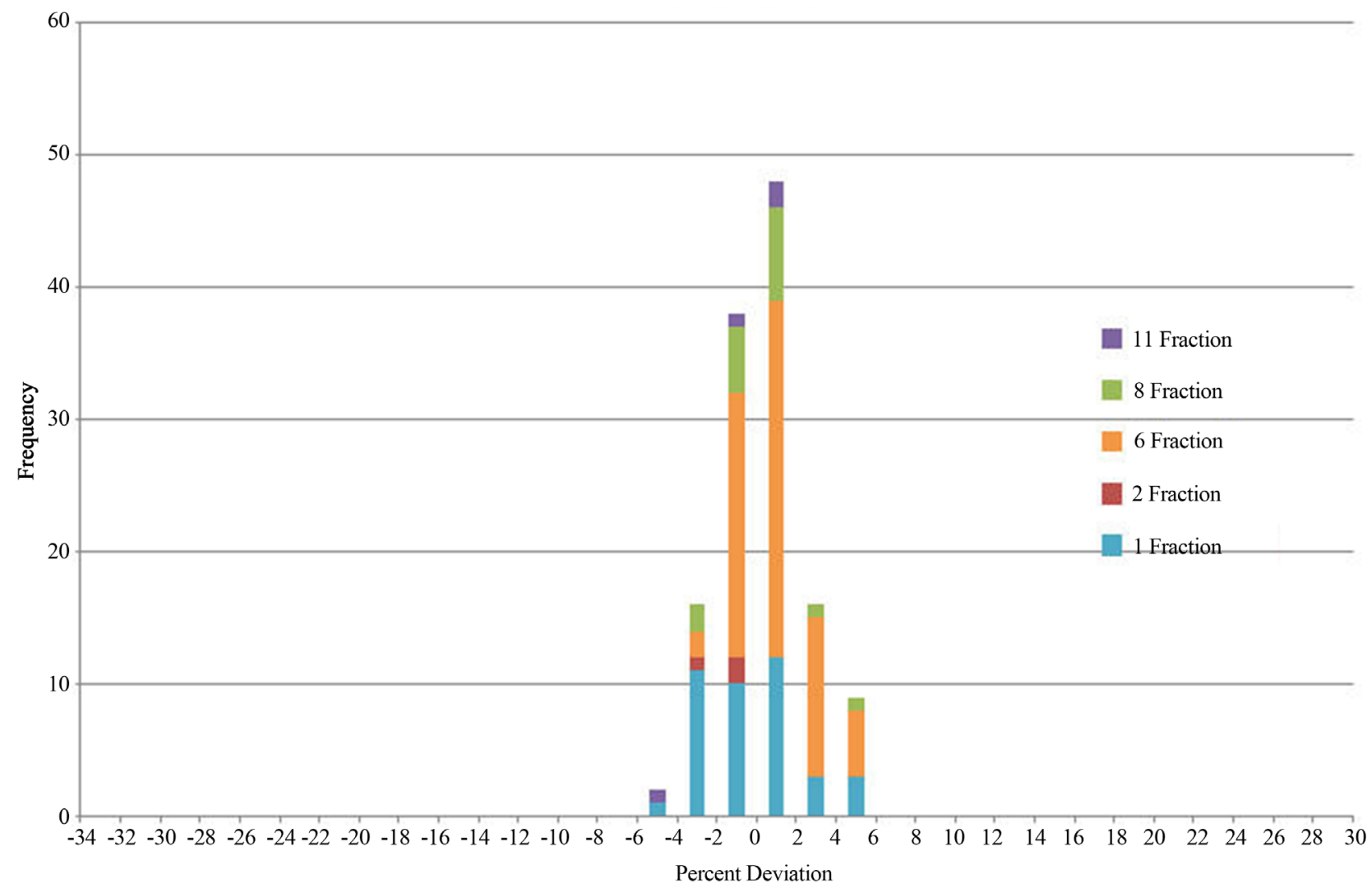



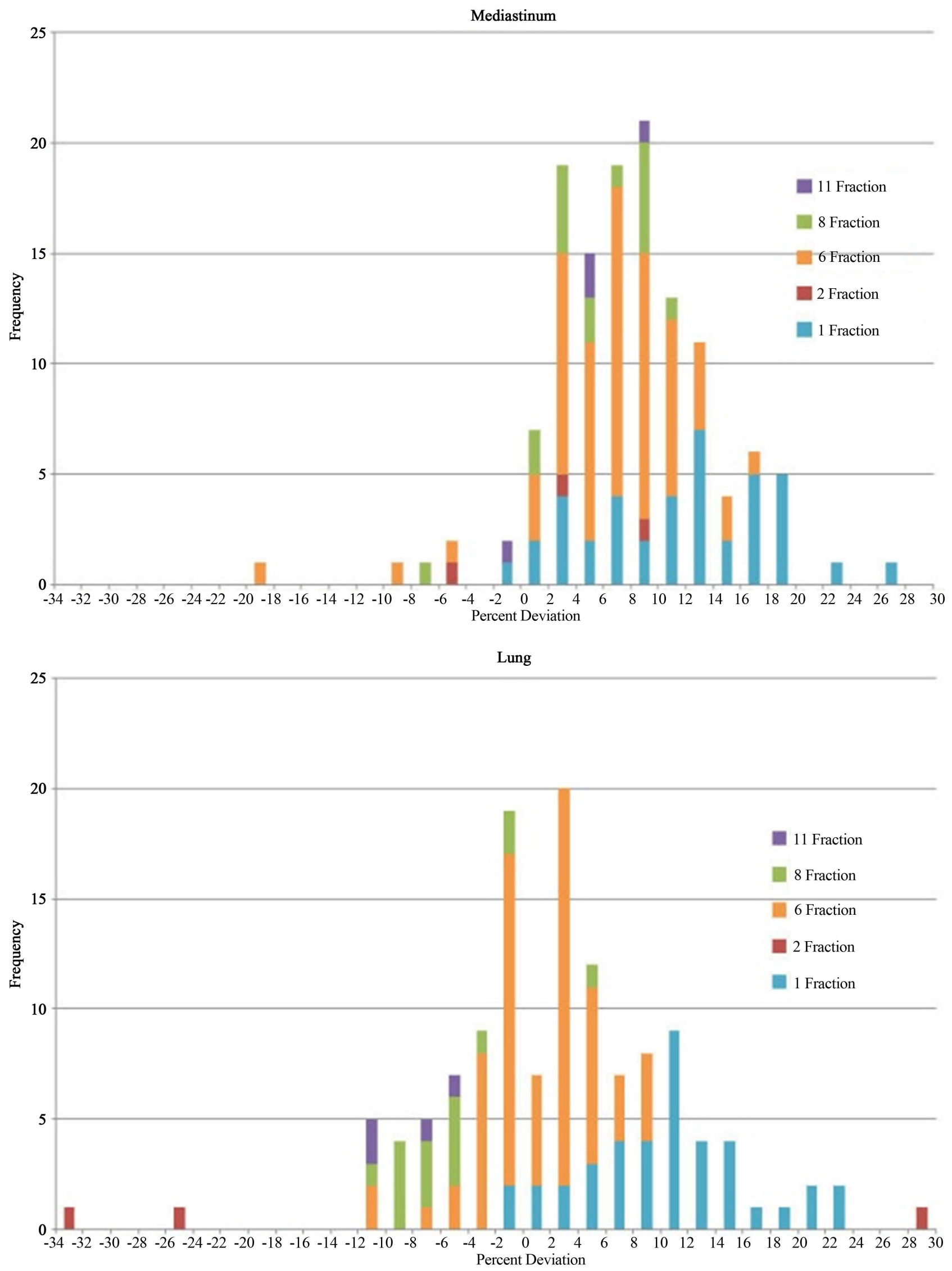

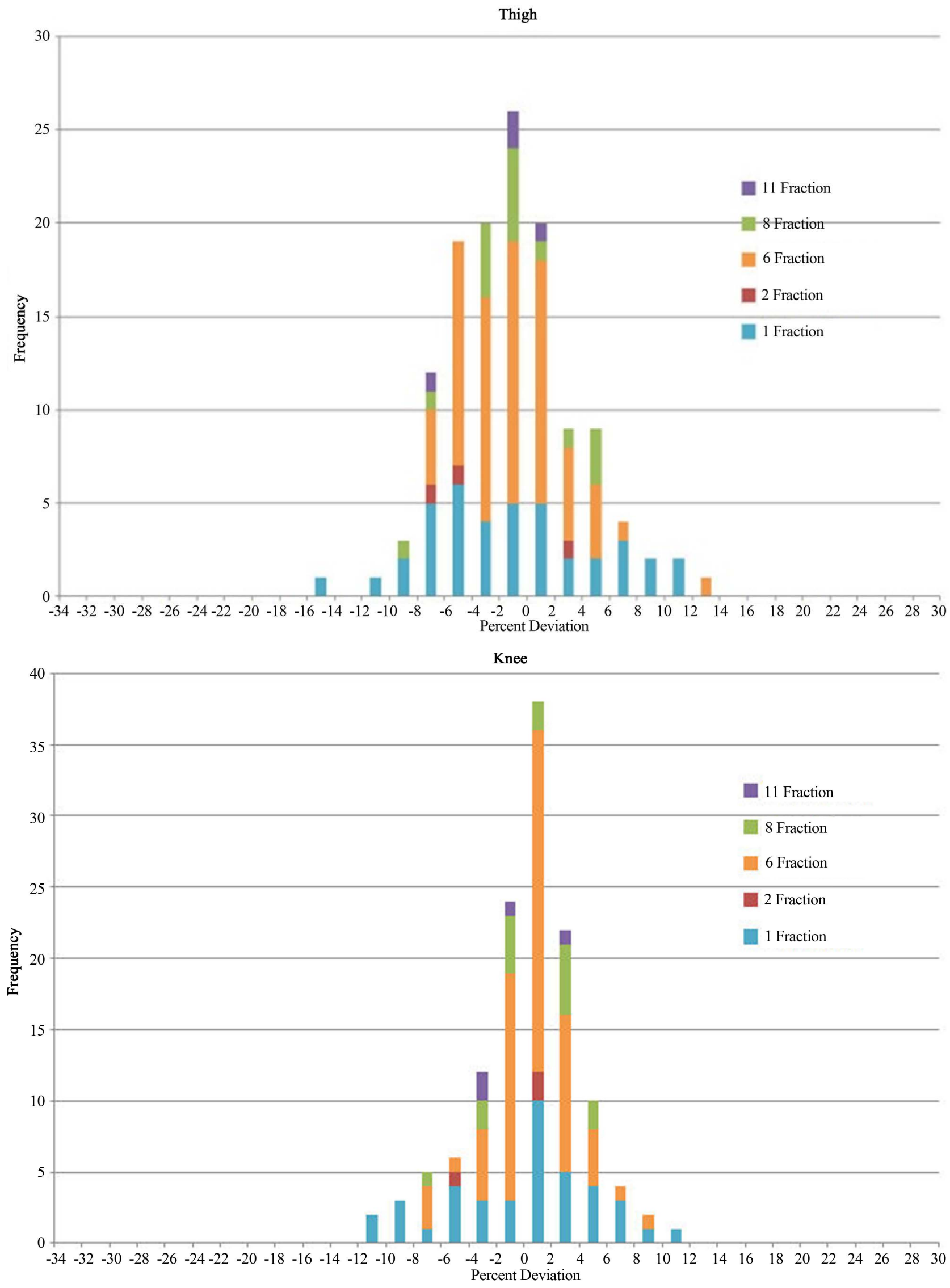


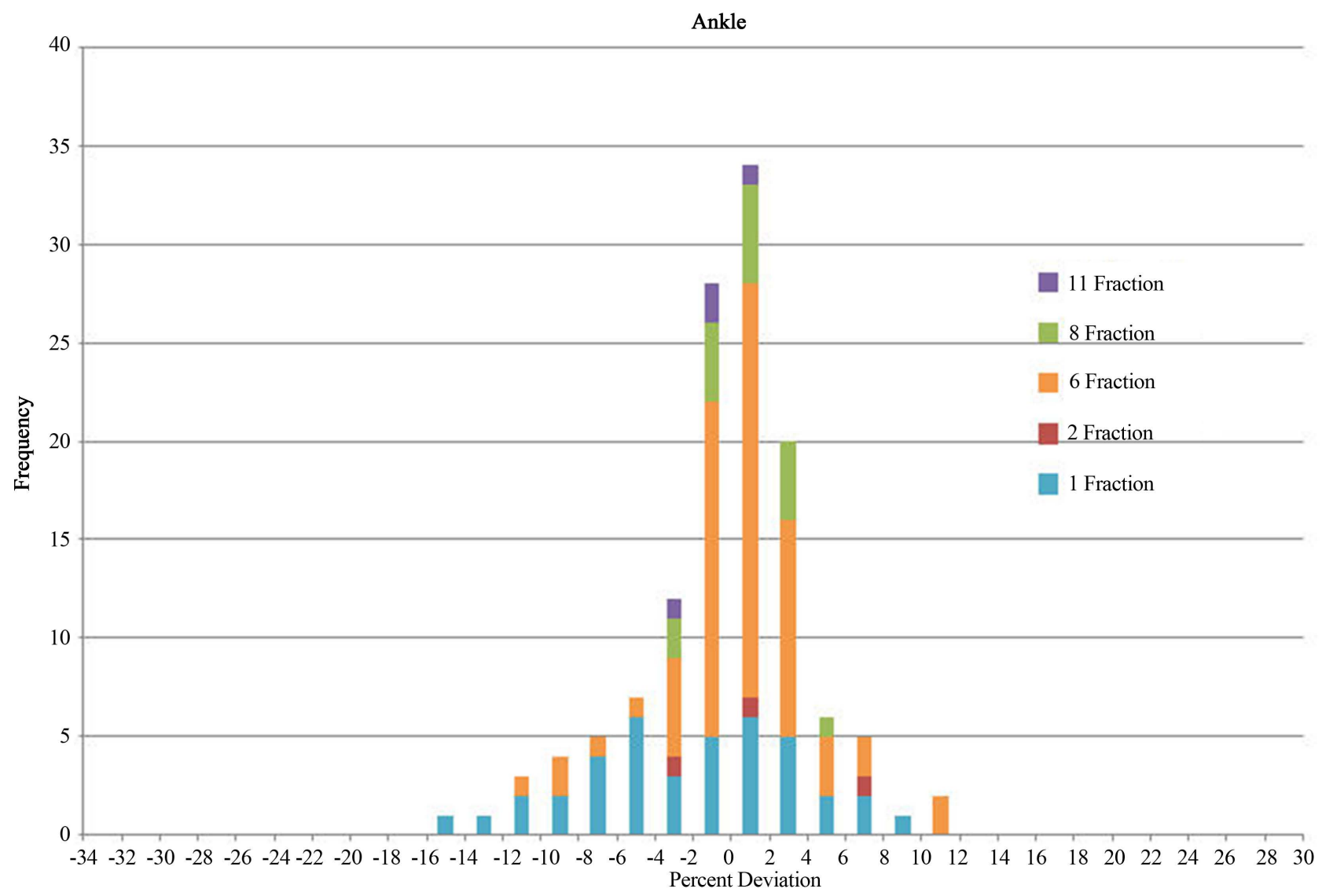

Figure 2. Histograms of dose deviation from prescribed umbilicus dose in \% for different body parts in all types of TBI treatments.

for the arm and mediastinum, for instance, where the dose deviation is larger compared with other body parts. For most histograms, the peaks are near the center at zero. For the mediastinum, because the lung blocks were so close to the region, we generally could not use compensators for the mediastinum. The slightly larger dose deviation for the arm was due to the arm's special position for AP and PA fields. The dose deviation distribution for the umbilicus had the narrowest range compared with all other body parts, which indicated that umbilicus received the most accurate dose intended by the prescription. This is understandable because the MU calculation was based on the prescription to the midline of umbilicus. The range of dose deviation for each body part is shown in Table 3. Only "1-fraction" and "6-fraction" data have enough of a data pool to show statistically meaningful results. Maximum deviations are found in "Arm" and "Mediastinum". For example, for "6-fraction" data, the dose deviation range for "Arm" is $-17 \%$ to $13 \%$ and for "Mediastinum", $-19 \%$ to $17 \%$. The results also indicate that single-fraction treatment in general has the largest dose deviation for all body parts compared with multi-fraction treatment regimes. For a multi-fraction case, physicists had more opportunities to adjust compen- sator thickness during the treatment process according to the readings from online dosimetry monitors during the entire course of the treatment, but for a single-fraction treatment, the option to adjust was limited. As we didn't use lung blocks for single-fraction treatments, the dose to the lung was always larger than the dose to the umbilicus, and its dose deviation histogram is positive and offset from 0 .

\subsection{Dose Deviation versus Thickness of a Body Part}

Figure 3 shows dose deviation versus body part thickness for each body part. From these graphs, we don't see any dependence of the dose deviation on the body part thickness. This may indicate that compensators used in the treatments and the way of using them in general worked well for most treatments. The dose deviation on TBI treatments seems more likely to have come from the method itself-a simple AP/PA field manual MU calculation without image- and computer-based 3D treatment planning.

From the above retrospective dosimetry study of the 129 TBI patient treatments, we found that for most of the 

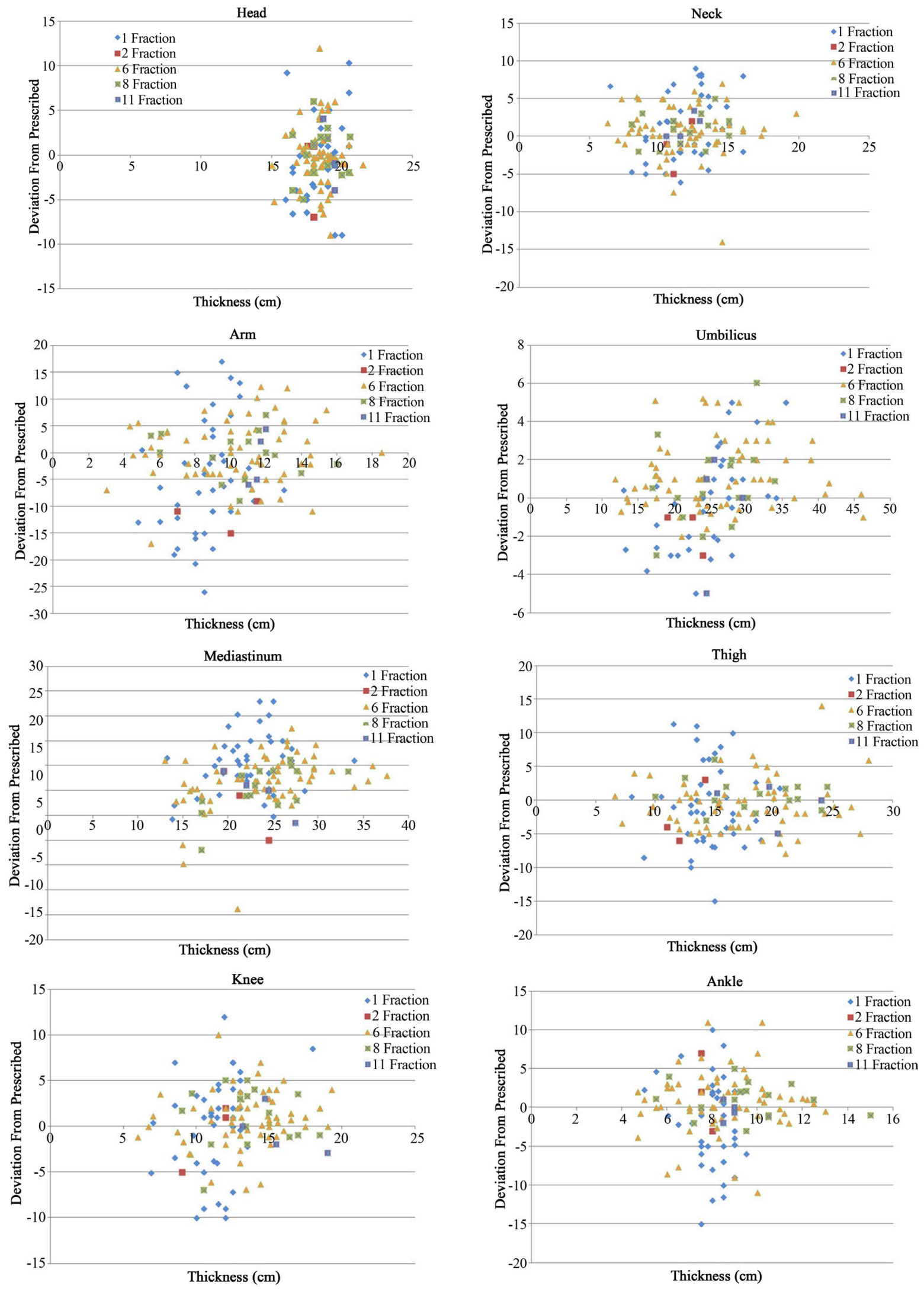

Figure 3. Dose deviations from prescribed umbilicus dose in \% versus thickness for different body parts in all types of TBI treatments. 
Table 3. Ranges of dose deviation for different body parts.

\begin{tabular}{cccccc}
\hline Dose deviation (\%) & 1-fraction & 2-fraction & 6-fraction & 8-fraction & 11-fraction \\
\hline Head & $-9-11$ & $-7-1$ & $-9-11$ & $-5-5$ & $-5-3$ \\
Neck & $-7-9$ & $-5-1$ & $-15-7$ & $-3-5$ & $-1-3$ \\
Arm & $-27-17$ & $-15--9$ & $-17-13$ & $-9-7$ & $-7-5$ \\
Umbilicus & $-5-5$ & $-3--1$ & $-3-5$ & $-3-5$ & $-5-1$ \\
Mediastinum & $-1-27$ & $-5-9$ & $-19-17$ & $-7-11$ & $-1-9$ \\
Lung & $-1-23$ & $-33-29$ & $-11-9$ & $-11-5$ & $-11--5$ \\
Thigh & $-15-11$ & $-7-3$ & $-7-13$ & $-9-5$ & $-7-1$ \\
Knee & $-11-11$ & $-5-1$ & $-7-9$ & $-7-5$ & $-3-3$ \\
Ankle & $-15-9$ & $-3-7$ & $-11-11$ & $-3-5$ & -3 \\
\hline
\end{tabular}

patients, the doses for different body parts were within the window of $10 \%$ difference from the prescribed dose. This meets the goal of our quality management program for TBI treatments. For the body parts we monitored with in vivo dosimetry, the dose was quite uniform in general and the peaks of histograms are centered near zero. However, the dose deviation in many histograms ranging from about $-10 \%$ to $10 \%$ indicate some incongruity of dose distribution. This could be due to the method of using lead compensators for a manual dose adjustment which could not ideally compensate for different body thicknesses everywhere.

\section{Discussion}

Although our results indicate that the conventional TBI can reach the main dosimetry goal in clinic, there are still some disadvantages of this method. From our experience, each fraction of TBI treatment with lung blocks usually takes more than an hour. Much of the time is consumed in placing and adjusting lung block positions based on the patient setup computerized radiograph result and the thickness of compensators based on dose monitoring results. This does not even account for the factor of special situations such as a patient's complications due to illness. The entire treatment procedure seems to be cumbersome and time consuming. Moreover, although the results from in vivo dosimetry indicate that the dose to the body parts being monitored is quite uniform, we still do not know the details of dose distribution for the body parts that are not under monitoring, especially for some critical organs. To use many dosimeters for in vivo dosimetry to monitor as many body parts is simply impractical. Furthermore, there were some uncertainties in diode measurements. One uncertainty could come from the diode itself-for example, the diode angular dependence to the direction of radiation beam could cause up to $10 \%$ difference [7]. Another uncertainty might come from the variations of patient's treatment position between different fractions because an immobilization device was not used.

For some TBI patients, boosting the dose to some regions of the body such as the bone marrow may benefit the patient. However, this will be difficult to accomplish in the conventional TBI. A CT image- and computerbased TBI treatment planning technique seems to be the method to solve the disadvantage of the conventional TBI, and this has been tried using tomotherapy technique [4-6]. A TBI clinical trial using tomotherapy has been going on [8]. However, for linac and image based TBI, due to the limitation in the capability of current linacs and the length of a normal patient, efforts still need to be made for implementation of the new technique. We have been trying to develop a new technique using intensity modulated radiation therapy for TBI and have obtained some preliminary results [9].

\section{Acknowledgements}

The authors are indebted to Dr. Nicholas Denko and Dr. Steven Devine for their helpful discussions. Jonathan Filippi also wants to thank Prof. Dehua Pei for being his chemistry research advisor which has allowed him to work on this research.

\section{REFERENCES}

[1] L. J. V. Dyk, et al., "The Physical Aspects of Total and Half Body Photon Irradiation,” AAPM Report No. 17 (Task Group 29), American Institute of Physics, College Park, 1986.

[2] F. M. Khan, "The Physics of Radiation Therapy," 4th Edition, Lippincott Williams \& Wilkins, Philadelphia, 2009, p. 405.

[3] R. Yao, D. Bernard, J. Turian, et al., “A Simplified Technique for Delivering Total Body Irradiation (TBI) with Improved Dose Homogeneity,” Medical Physics, Vol. 39, 
No. 4, 2012, pp. 2239-2248.

http://dx.doi.org/10.1118/1.3697526

[4] J. Y. Wong, A. Liu, T. Schultheiss, et al., "Targeted Total Marrow Irradiation Using Three-Dimensional ImageGuided Tomographic Intensity-Modulated Radiation Therapy: An Alternative to Standard Total Body Irradiation,” Biology of Blood and Marrow Transplantation, Vol. 12, No. 3, 2006, pp. 306-315. http://dx.doi.org/10.1016/j.bbmt.2005.10.026

[5] S. K. Hui, J. Kapatoes, J. Fowler, et al., "Feasibility Study of Helical Tomotherapy for Total Body or Total Marrow Irradiation,” Medical Physics, Vol. 32, No. 10, 2005, pp. 3214-3224. http://dx.doi.org/10.1118/1.2044428

[6] B. Aydogan, A. J. Mundt and J. C. Roeske, "Linac-Based Intensity Modulated Total Marrow Irradiation (IM-TMI),"
Technology in Cancer Research and Treatment, Vol. 5, No. 5, 2006, pp. 513-519.

[7] P. A. Jursinic, "Angular Dependence of Dose Sensitivity of Surface Diodes,” Medical Physics, Vol. 36, No. 6, 2009, pp. 2165-2171. http://dx.doi.org/10.1118/1.3125644

[8] A. S. Stein, "Intensity-Modulated Radiation Therapy, Etoposide, and Cyclophosphamide Followed by Donor Stem Cell Transplant in Treating Patients With Relapsed or Refractory Acute Lymphoblastic Leukemia,” Clinical Trials.gov Identifier: NCT00576979, 2013.

[9] D. V. BonCoeur, C. Pelloski, N. Gupta, et al., "Using Intensity Modulated Radiation Therapy for Total Body Irradiation-A Phantom Study," Medical Physics, Vol. 38, 2011, p. 3678. http://dx.doi.org/10.1118/1.3612777 